\title{
EMOCIONES QUE EXPERIMENTAN LOS MAESTROS EN FORMACIÓN HACIA LAS MATEMÁTICAS
}

\section{EMOTIONS EXPERIENCED BY TRAINEE TEACHERS TOWARDS MATHEMATICS}

\author{
Raúl Prada Núñez ${ }^{1}$ \\ César Augusto Hernández Suárez² \\ William Rodrigo Avendaño Castro ${ }^{3}$
}

\section{RESUMEN}

El artículo muestra los hallazgos de un estudio cuantitativo - descriptivo transversal que buscó identificar las emociones que experimentan los futuros docentes de Matemáticas durante su proceso de formación profesional. Se utilizó un cuestionario tipo Likert con cinco niveles de respuesta, el cual fue aplicado a una muestra de 198 estudiantes matriculados durante el primer semestre del 2020 en el programa de Licenciatura

$1 \quad$ Magíster en Ingeniería de Análisis de Datos, Mejora de Procesos y Toma de Decisiones por la Universidad Politécnica de Valencia (España). Docente investigador de la Universidad Francisco de Paula Santander. Correo electrónico: raulprada@ufps.edu.co. Orcid: 0000-0001-61451786.

$2 \quad$ Magister en Educación Matemática por la Universidad Nacional Experimental del Táchira (Venezuela). Docente investigador de la Universidad Francisco de Paula Santander. Correo electrónico: cesaraugusto@ufps.edu.co. Orcid: 0000-0002-7974-5560.

3 Doctor en Ciencias Sociales y Humanas por la Pontificia Universidad Javeriana. Docente investigador de la Universidad Francisco de Paula Santander. Correo electrónico: williamavendano@ufps.edu.co. Orcid: 0000-00027510-8222. en Matemáticas en una universidad pública del nororiente colombiano. Los resultados permiten identificar que el $61 \%$ de los participantes tienen una percepción positiva respecto a los procesos de enseñanza y aprendizaje de las Matemáticas, puesto que reconocen que para triunfar en la asignatura se requiere de interés, seguridad, perseverancia y tranquilidad en la resolución de ejercicios o problemas matemáticos. Se concluye que estos sentimientos son más fuertes en las mujeres, pero sin diferencias significativas entre semestres académicos cursados o por edad.

PALABRAS CLAVE: emociones hacia las matemáticas, maestros en formación, educación superior

\section{ABSTRACT}

The article shows the findings of a cross-sectional quantitative-descriptive study that sought to identify the emotions that future mathematics 
teachers experience during their professional training process. A Likert-type questionnaire with five response levels was used, which was applied to a sample of 198 students enrolled during the first semester of 2020 in the Bachelor of Mathematics program at a public university in northeastern Colombia). The results allow identifying that $61 \%$ of the participants have a positive perception regarding the teaching and learning processes of Mathematics, since they recognize that to succeed in the subject requires interest, security, perseverance and tranquility in the resolution of mathematical exercises or problems. It is concluded that these feelings are stronger in women, but without significant differences between academic semesters or age.

KEYWORDS: Emotions towards mathematics, future teachers, higher education.

\section{INTRODUCCIÓN}

Diversas investigaciones se han desarrollado desde la Educación Matemática, muchas de ellas centradas en determinar las dificultades en los procesos de enseñanza y aprendizaje de esta área como los trabajos de Riviere (1990), Rico (1995), Kilpatrick, Gómez y Rico (1998), González-Pineda y Álvarez (1998), Godino, Batanero y Font (2003), Orrantia (2006), Rebollo y Rodríguez (2006), Socas (2007), entre muchos otros; pero las dificultades siguen presentes, luego parecieran infructuosos las investigaciones.

Algunos trabajos como el de Araya (2007), Pichardo y Puente (2012) o Vargas, Vega y Morales (2020), han recurrido al uso de recursos TIC en el proceso de enseñanza de las Matemáticas, concluyendo que se logra mejorar la motivación, pero aún siguen persistiendo un buen número de dificultades que impiden una correcta apropiación de los conceptos matemáticos.
En Prada, Gamboa y Avendaño (2020), se afirma que el origen de las dificultades de aprendizaje junto con la aversión hacia la asignatura están propiciadas por una amplia diversidad de factores, algunos asociados con la naturaleza abstracta del saber, mientras que otros son el resultado de la interacción social en la casa o en la escuela, lo que termina generando una buena cantidad de prejuicios con respecto a la actividad matemática y ello incide de forma directa en el proceso de enseñanza y aprendizaje. En la investigación de Gil, Blanco y Guerrero (2006) se confirma la influencia que tienen la familia, el círculo de amistades y los docentes en la generación de sentimientos de angustia y ansiedad que propician en el estudiante una actitud de rechazo respecto de las matemáticas.

La afectividad y las emociones han sido ampliamente estudiados desde la psicología, pero desde los años 90 se empezó a prestar atención a la relación entre las emociones y la educación (Sutton \& Wheatley, 2003). Frijda (2000) afirma que emoción y cognición están interconectados y a la vez difícil de separar, por ello Marina (2004) afirma que "las emociones influyen en el conocimiento, pero el conocimiento influye en las emociones" (p. 53). Para Costillo, Borrachero, Brígido y Mellado (2013) "lo cognitivo configura lo afectivo y lo afectivo condiciones los cognitivo" (p. 515).

En las últimas décadas, a partir de los trabajos pioneros de McLeod (1989, 1992, 1994), Gómez (1997, 2000, 2003), Gil, Blanco y Guerrero (2005) y Martínez (2005) se ha investigado el efecto que tiene la dimensión afectiva en el proceso de aprendizaje de las Matemáticas. Gil, blanco y Guerrero (2005) afirman que el dominio afectivo hacia las Matemáticas está compuesto por creencias, la cuales permiten a las personas a organizar y filtrar la información que percibe del entorno para ir elaborando su propia noción de la realidad (Gómez, 2000), las actitudes, vistas como la predisposición evaluativa de 
la conducta que determina las intenciones personales e influye en el comportamiento (Giménez y Flores, 2017) y las emociones, las cuales son respuestas afectivas fuertes que no son sólo automáticas sino que son el resultado del aprendizaje, de la influencia social y de la interpretación (Martínez , 2014).

Autores como Hargreaves (2000) y Ritchie, Tobin, Hudson, Roth \& Mergard (2011) señalan que la enseñanza se considera una práctica emocional, donde intervienen tanto la razón como la pasión (Cochran, 2003). Otero (2006) señala que no hay actividad del ser humano, sin una emoción que la haga posible y la fundamente; es por ello que el objetivo de esta investigación es explorar las emociones que experimentan los futuros docentes en Matemáticas durante su proceso de formación académica en la universidad. Este estudio al igual que los trabajos de Melero (2000), Alzina (2005), Domínguez, Pescador y Melero (2009), de Nicolás, Torremorell y Valls (2016), Cejudo, López, Rubio y Latorre (2015), intenta aportar a la comprensión y la explicación de las emociones de los futuros maestros sobre el área de su disciplina de formación .

\section{METODOLOGÍA}

Los participantes de esta investigación corresponden a un grupo de 212 estudiantes que para el primer semestre del 2020 estaban matriculados en el programa de Licenciatura en Matemáticas de una población conformada por 342 estudiantes, en una institución de educación superior de carácter público. Este programa se encuentra adscrito a la Facultad de Educación y forma parte de una amplia oferta académica en la que hay tres programas más de formación en maestros. Los participantes diligenciaron un cuestionario anónimo de forma voluntaria sobre dos aspectos principales, inicialmente una descripción demográfica seguida de una sección en la que se caracterizaron los descriptores básicos del dominio afectivo (creencias, actitudes y emociones) hacía las Matemáticas.
Este informe se concentra en el análisis de las características demográficas y las emociones que experimentan estas personas en su trabajo diario con las Matemáticas. En Prada, Marmolejo y Mulema (2020) se hace uso de un cuestionario que combina elementos de diversos autores, que para el caso de las emociones se toma como referencia el cuestionario propuesto por Fernández-Cézar et al. (2016) que surge como una modificación del cuestionario de Auzmendi (1992) que contiene 10 ítems.

El grado de conformidad con cada ítem lo evalúa el participante mediante el uso de una escala Likert con cinco niveles de respuesta: a) dos con tendencia negativa reflejado en las calificaciones 1 (Muy en desacuerdo) y 2 (En desacuerdo); b) un nivel intermedio con calificación 3 (Indiferente); c) dos con tendencia positiva reflejado con las calificaciones 4 (De acuerdo) y 5 (Muy de acuerdo). El cuestionario fue validado tanto por juicio de expertos como mediante la prueba Alfa de Cronbach arrojando un coeficiente de 0,798 para el caso de las emociones, valor que resulta admisible según la literatura sugerida (Prada, Marmolejo y Mulema, 2020).

El cuestionario fue auto diligenciado por cada participante, el cual recibió un correo en el que se explicaba el objetivo de la investigación y se invitaba a su cumplimentación por medio del acceso a un link. Los datos fueron recolectados durante el mes de mayo del 2020 y posteriormente fueron descargados como archivo de Excel del Google Form y después de un proceso de revisión y validez de las respuestas fueron exportados al SPSS v25 para ser procesados y analizados de forma descriptiva.

Por todo lo anteriormente, se concluye que este proceso investigativo se ajusta al enfoque cuantitativo, a nivel descriptivo transversal y con diseño de campo, puesto que los datos fueron recolectados en un momento específico del tiempo directamente de la fuente primaria, 
sin manipulación de las variables por parte del investigador y bajo la aplicación de un muestreo no probabilístico (Prada, Gamboa y Hermández, 2020).

\section{RESULTADOS Y DISCUSIÓN}

\section{Características demográficas de los participantes}

En la Tabla 1 se muestran las características demográficas de los participantes, dentro de las que se destaca: el predominio del género masculino, aproximadamente el $91 \%$ tienen edades que oscilan entre 15 y 25 años; en cuanto al semestre académico que cursa, se observa participación de todos los semestres de la propuesta curricular del programa, aunque en el segundo y sexto semestre fue inferior con respecto a los demás semestres.

Tabla 1

Descripción de las características demográficas de los informantes

\begin{tabular}{|c|c|c|c|}
\hline Variable & Opciones de respuesta & Frecuencia & Porcentaje \\
\hline \multirow{3}{*}{ Género } & Mujer & 108 & 54,7 \\
\hline & Hombre & 90 & 45,3 \\
\hline & Total & 198 & 100,0 \\
\hline \multirow{5}{*}{ Edad } & De 15 a 20 años & 90 & 45,3 \\
\hline & De 21 a 25 años & 90 & 45,3 \\
\hline & De 26 a 30 años & 16 & 8,0 \\
\hline & 31 años o más & 2 & 1,4 \\
\hline & Total & 198 & 100,0 \\
\hline \multirow{11}{*}{$\begin{array}{c}\text { Semestre académico } \\
\text { que cursa }\end{array}$} & Primero & 16 & 8,0 \\
\hline & Segundo & 6 & 3,3 \\
\hline & Tercero & 36 & 17,9 \\
\hline & Cuarto & 20 & 9,9 \\
\hline & Quinto & 28 & 14,2 \\
\hline & Sexto & 6 & 3,3 \\
\hline & Séptimo & 26 & 13,2 \\
\hline & Octavo & 17 & 8,5 \\
\hline & Noveno & 29 & 14,6 \\
\hline & Décimo & 14 & 7,1 \\
\hline & Total & 198 & 100,0 \\
\hline
\end{tabular}

Fuente: Elaboración propia

\section{EMOCIONES HACIA LAS MATEMÁTICAS}

Con el fin de simplificar la interpretación de los resultados, se realiza un ajuste en la escala valorativa así: las opciones de respuesta totalmente en desacuerdo y en desacuerdo se agruparon en una categoría llamada rechaza; el indiferente se mantiene igual y las opciones de acuerdo y totalmente de acuerdo se agrupan en la categoría acepta. El gráfico1 permite evidenciar que aproximadamente el $61 \%$ de los participantes manifiestan aceptación ante cada uno de los ítems mencionados en esta sección del cuestionario. Se reconoce como un elemento de análisis, que aproximadamente el $24 \%$ de los informantes manifiestan rechazo ante las emociones analizadas. 


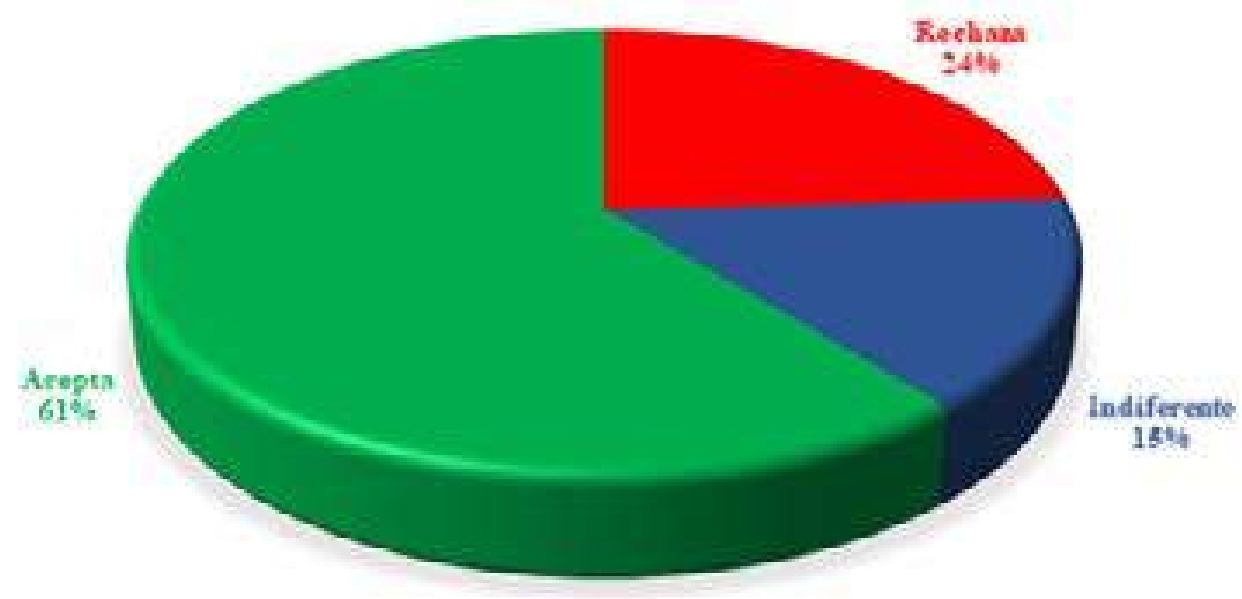

Gráfico 1. Percepción promedio sobre los ítems asociados a las Emociones hacia las Matemáticas en opinión de los participantes

Apartir de la información mostrada anteriormente, es importante señalar aquellos aspectos en los cuales los participantes muestran mayor aceptación y/o rechazo. En este sentido, a través de la Tabla 2 se puede identificar que los participantes están de acuerdo con aquellos ítems en dónde se asocia el interés, la curiosidad, la seguridad, la felicidad, el esfuerzo, la perseverancia, la paciencia y la tranquilidad al trabajo matemático sin importar si llega a la solución correcta o si debe intentarlo varias veces. En lo que respecta a aquellos ítems que fueron rechazados por los participantes, se destacan aquellos en dónde se asume una postura de inconstancia, inseguridad, fracaso o ansiedad al resolver ejercicios y/o problemas matemáticos.

Tabla 2

Resultados de la valoración de los ítems en opinión de los participantes

\begin{tabular}{|l|c|c|c|c|}
\hline \multicolumn{2}{|c|}{ Ítems } & Rechaza & Indiferente & Acepta \\
\hline E1. Me rindo fácilmente cuando me piden resolver & Frec. & 158 & 30 & 10 \\
\cline { 2 - 5 } $\begin{array}{l}\text { un ejercicio en Matemáticas, incluso sin encontrar la } \\
\text { solución }\end{array}$ & $\%$ & 79,7 & 15,1 & 5,1 \\
\hline $\begin{array}{l}\text { E2. Siento curiosidad por conocer la respuesta } \\
\text { cuando el profesor me pide que resuelva un ejercicio } \\
\text { en Matemáticas }\end{array}$ & Frec. & 14 & 17 & 167 \\
\cline { 2 - 5 } & $\%$ & 7,1 & 8,5 & 84,4 \\
\hline $\begin{array}{l}\text { E3. Me siento nervioso cuando el profesor me pide } \\
\text { por sorpresa que resuelva en el tablero un ejercicio } \\
\text { de Matemáticas }\end{array}$ & Frec. & 52 & 56 & 90 \\
\cline { 2 - 5 } & $\%$ & 26,5 & 28,3 & 45,3 \\
\hline $\begin{array}{l}\text { E4. Cuando resuelvo ejercicios de Matemáticas en } \\
\text { grupo, me siento más seguro }\end{array}$ & Frec. & 28 & 42 & 128 \\
\cline { 2 - 6 } & $\%$ & 14,2 & 21,2 & 64,7 \\
\hline $\begin{array}{l}\text { E5. Cuando no me sale la solución de un ejercicio de } \\
\text { Matemáticas empiezo a sentirme inseguro, ansioso } \\
\text { y nervioso }\end{array}$ & Frec. & 74 & 48 & 76 \\
\cline { 2 - 6 } & $\%$ & 37,2 & 24,5 & 38,2 \\
\hline
\end{tabular}




\begin{tabular}{|l|c|c|c|c|}
\hline E6. Si no encuentro la solución de un ejercicio en & Frec. & 104 & 46 & 48 \\
\cline { 2 - 5 } $\begin{array}{l}\text { Matemáticas, tengo la sensación de haber fracasado } \\
\text { y de haber perdido el tiempo }\end{array}$ & $\%$ & 52,4 & 23,1 & 24,5 \\
\hline \multirow{2}{*}{$\begin{array}{l}\text { E7. Me siento feliz cuando resuelvo correctamente } \\
\text { un ejercicio en Matemáticas }\end{array}$} & Frec. & 11 & 4 & 183 \\
\cline { 2 - 5 } & $\%$ & 5,7 & 1,9 & 92,4 \\
\hline \multirow{2}{*}{$\begin{array}{l}\text { E8. Cuando fallo al intentar resolver un ejercicio en } \\
\text { Matemáticas, lo vuelvo a intentar, pero utilizando otro } \\
\text { método de solución }\end{array}$} & Frec. & 18 & 10 & 170 \\
\cline { 2 - 5 } & $\%$ & 9,0 & 5,2 & 85,9 \\
\hline \multirow{2}{*}{$\begin{array}{l}\text { E9. La resolución de un ejercicio en Matemáticas } \\
\text { exige esfuerzo, perseverancia y paciencia }\end{array}$} & Frec. & 11 & 10 & 177 \\
\cline { 2 - 5 } & $\%$ & 5,7 & 5,2 & 89,1 \\
\hline \multirow{2}{*}{$\begin{array}{l}\text { E10. Estoy calmado y tranquilo cuando resuelvo } \\
\text { ejercicios de Matemáticas }\end{array}$} & Frec. & 16 & 33 & 149 \\
\cline { 2 - 5 } & $\%$ & 8,4 & 16,5 & 75,1 \\
\hline \multirow{2}{*}{ Promedio } & Frec. & 48 & 30 & 120 \\
\cline { 2 - 5 } & $\%$ & 24,3 & 15,0 & 60,7 \\
\hline
\end{tabular}

Los resultados de este estudio se pueden contrastar con investigaciones como las de Castro y Miranda (2019); Ramírez (2019); Velazquez, Maldonado, Del Valle y Valdéz (2020), quienes señalan que la motiviación al estudio de las matemáticas se relaciona con la exigencia intelectual de está área del conocimiento, el reconocimiento público por parte de los profesores y la satisfacción personal de resolver problemas complejos.

Por otra parte, el rechazo a las matemáticas y los determinantes de este rechazo encontrados en el estudio, se pueden explicar desde el trabajo de Cerda et al.,(2016) quienes afirman que la inseguridad, el miedo al fracaso y el nerviosismo, constituyen predisposicines negativas que gereran barreras en todo proceso de aprendizaje, no solo de la matemática si no de otras áreas de conocimiento, pero por lo general son más marcadas en esta área.

\section{ANÁLISIS DE LAS EMOCIONES HACIA LAS MATEMÁTICAS DESAGREGADAS POR GÉNERO}

Como se mencionó los participantes aceptaron y rechazaron algunos ítems asociados con las emociones. Por medio del gráfico $2 a \mathrm{y}$ $2 \mathrm{~b}$, se analizan las diferencias por género en ambos aspectos. Con respecto a los ítems que presentaron un porcentaje de aceptación superior al $50 \%$, se pudo identificar que en cinco de ellos las mujeres superan en porcentajes a los hombres y de ellos en los ítems E2, E8 y E4 la diferencia oscila entre el $17 \%$ y el $21 \%$; es decir, que las mujeres participantes manifiestan una mejor actitud hacia las Matemáticas. 


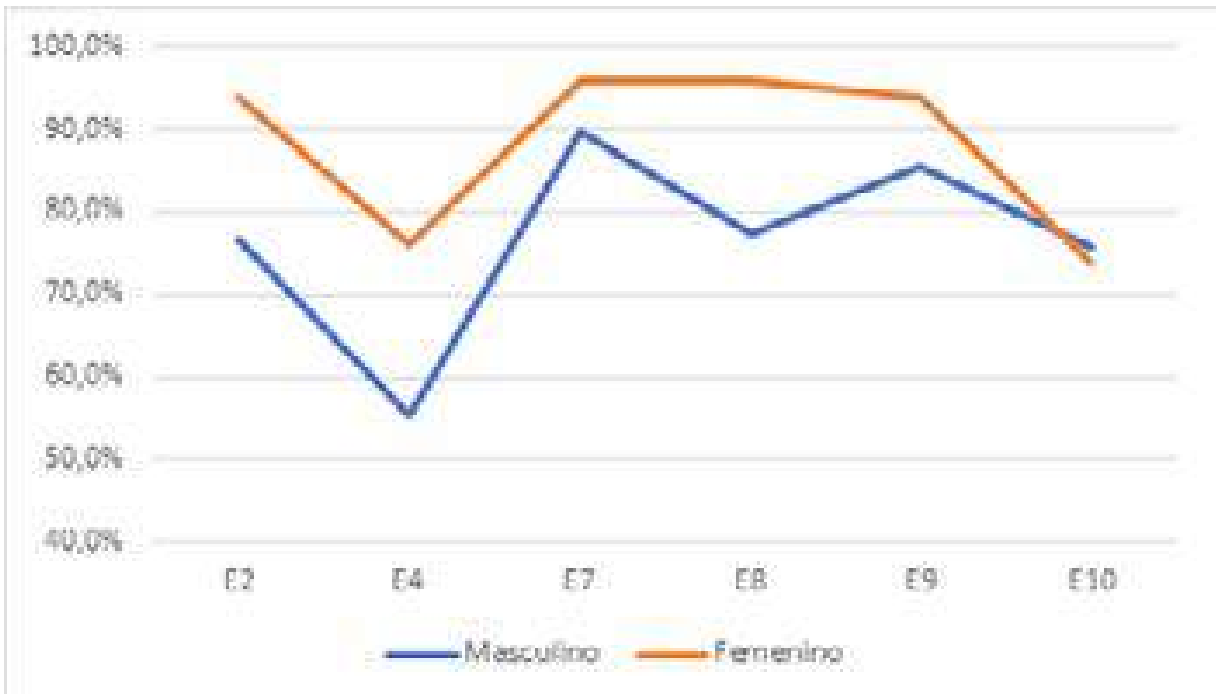

Gráfico 2a. Porcentaje de ítems aceptados por género de los participantes

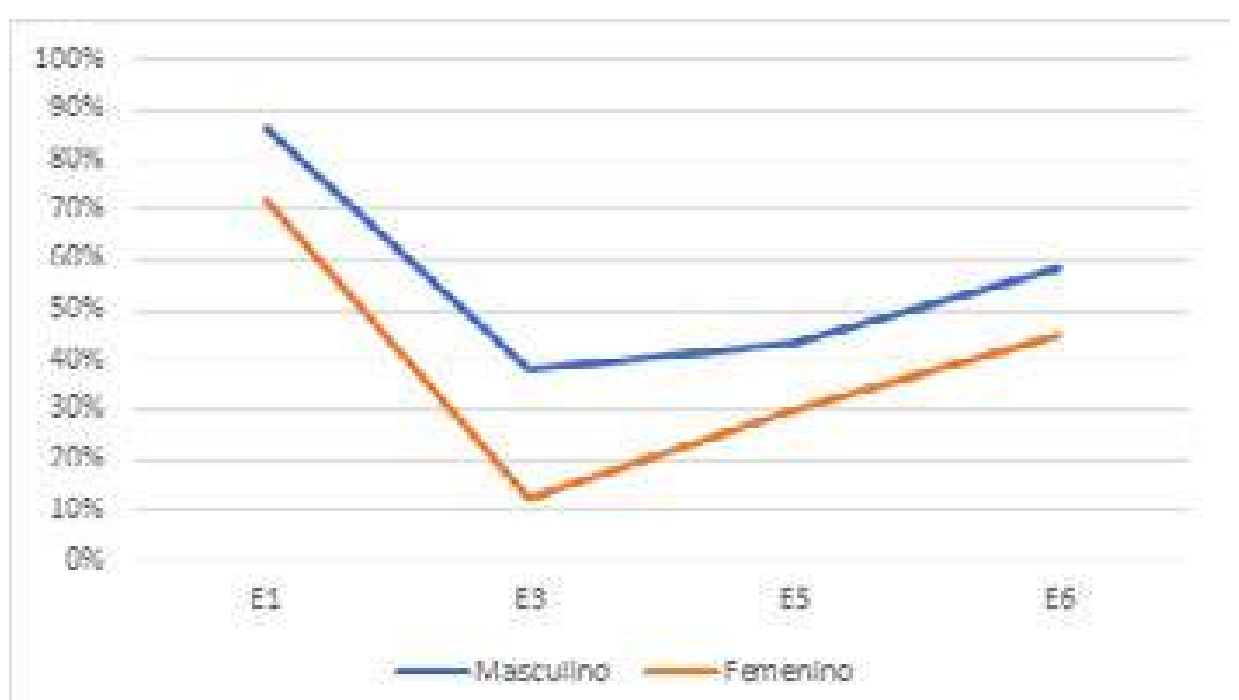

Gráfico $\mathbf{2 b}$. Porcentaje de ítems rechazados por género de los participantes

En cuanto al comportamiento por género respecto a aquellos ítems en dónde se mencionaban posturas de inconstancia, inseguridad, fracaso o ansiedad al resolver ejercicios y/o problemas matemáticos, se pudo determinar que el porcentaje de hombres que rechazan estas posturas es mucho mayor que el de las mujeres en al menos el $13 \%$ y no mayor al $26 \%$.

Los anteriores datos se pueden reconfirmar con estudios como el de Barbero, Holgado, Vila y Chacón (2007) quienes manifiestan que las mujeres tienen una tendencia importante a tener actitudes más positiva hacia la matemática que los hombres, esto debido a que las mujeres tinen unos mejores hábitos de estudio y por ende mejor rendimiento académico.

\section{ANÁLISIS DE LAS EMOCIONES HACIA LAS MATEMÁTICAS DESAGREGADAS POR CICLO DE FORMACIÓN}

Un elemento importante a analizar en el grupo de participantes, es identificar si su gusto por las Matemáticas cambia a medida que avanza en su proceso de formación académica como futuro Licenciado en Matemáticas. Para poder 
resolver esta duda, se aclara que dentro de la propuesta curricular del programa en estudio se han definido tres ciclos de formación distribuidos así: a) Ciclo de Fundamentación que corresponde a los semestres I, II y III; b) Ciclo de Especialización que corresponde a los semestres IV, V y VI; c) Ciclo de Profundización para los semestres VII, VIII, IX y X.

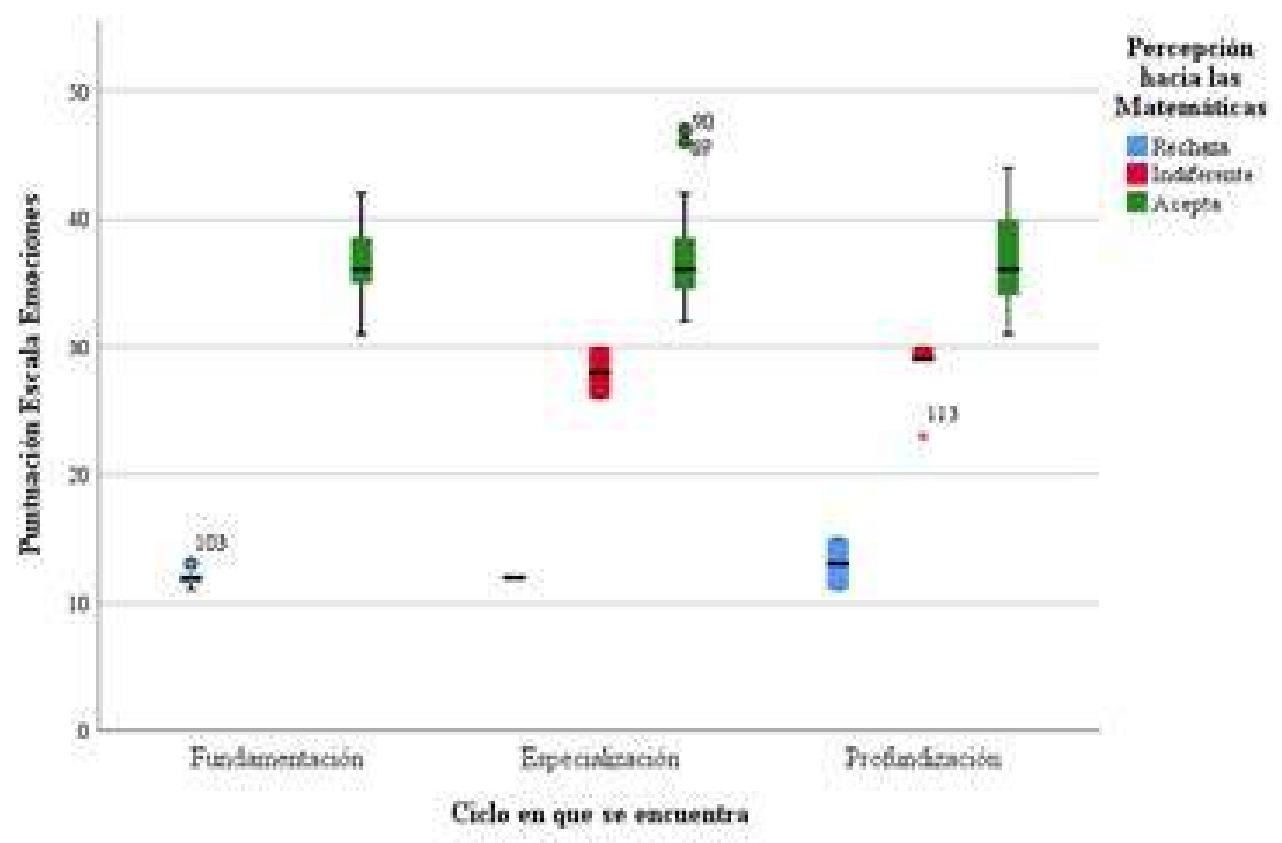

Gráfico 3. Diagrama de cajas y bigote de la percepción hacia las Matemáticas desagregada por según el ciclo de formación en que se encuentra el participante

Por medio del gráfico 3 se puede observar que, puesto que las cajas se solapan entre si según el ciclo de formación en cada uno de los diversos niveles de percepción hacia las Matemáticas, se concluye que el estudiante que ingresa al programa lo hace porque le gusta la asignatura y este gusto no cambia a medida que avanza semestre a semestre. En cuanto a la dispersión de las puntuaciones, se pudo observar que las opiniones de quienes tienen una percepción favorable hacia la asignatura son más diversas en los estudiantes del ciclo de profundización a pesar de tener la calificación media más alta de los tres ciclos.

\section{ANÁLISIS DE LAS EMOCIONES HACIA LAS MATEMÁTICAS SEGÚN GRUPO ETARIO}

Para finalizar el análisis de las emociones hacia las Matemáticas observadas en los participantes de esta investigación, se desea indagar si existen diferencias en cuanto a las opiniones de los participantes a nivel general respecto a la edad. En la gráfica 4 se muestra el diagrama de cajas simple el cual permite verificar que respecto a las puntuaciones en la escala de emociones evaluada no se observan diferencias significativas entres los diversos intervalos de edad; pero los participantes de 31 años o más si exhiben las puntuaciones de percepción más bajas en contraste con los demás grupos. 


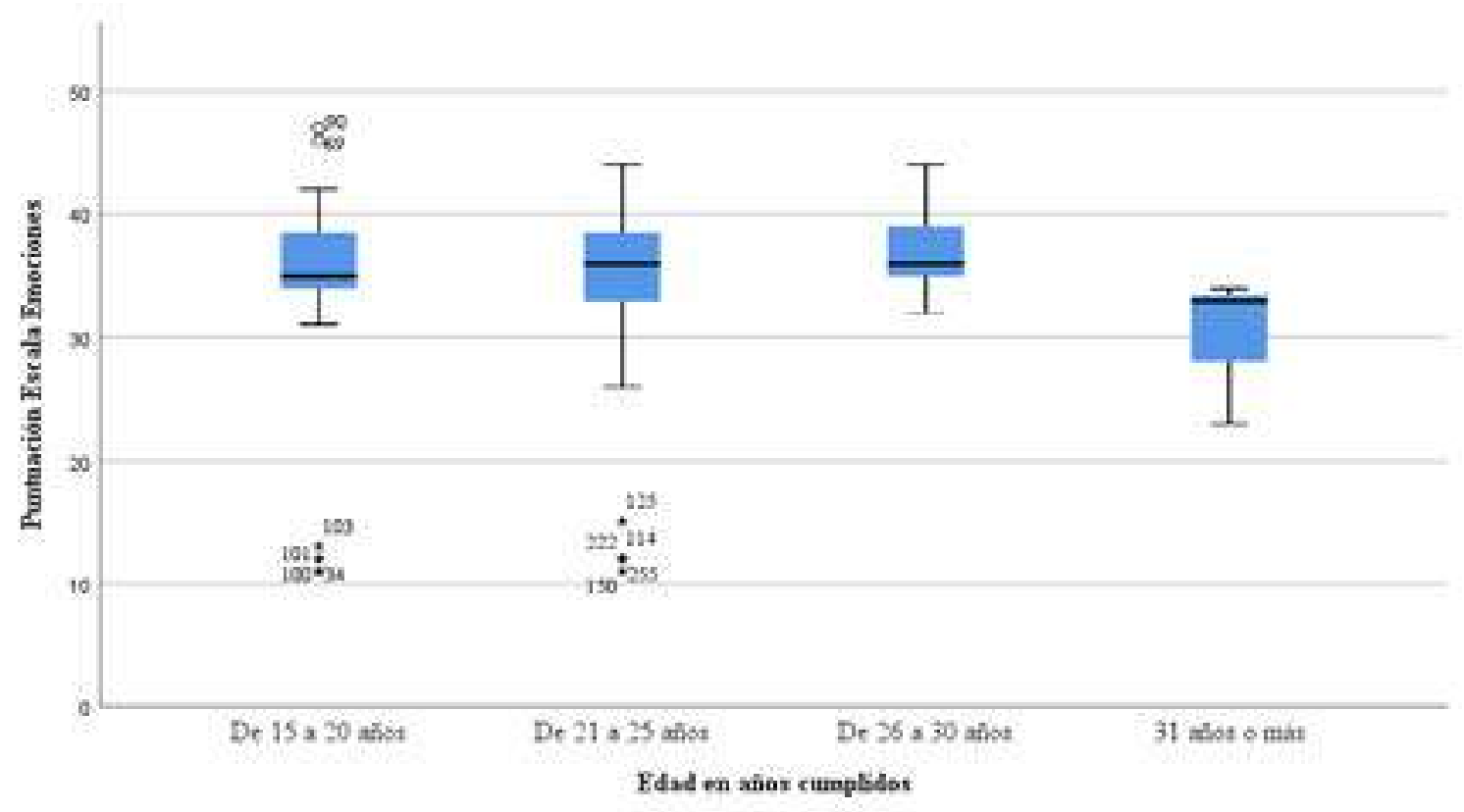

Gráfico 4. Diagrama de cajas simple de la percepción hacia las Matemáticas desagregada por el grupo etario en que se encuentra el participante

\section{CONCLUSIONES}

La información derivada del procesamiento de los datos proporcionados por los participantes permitieron concluir que los estudiantes vinculados al programa académico objeto de estudio, tienen una fuerte convicción de la importancia del conocimiento matemático.

Los maestros en formación reconocen que el conocimiento matemático es necesario para alcanzar el éxito en sus asignaturas y con sus actitudes de perseverancia buscan la solución de los problemas matemáticos y al tiempo proponen diversas alternativas de solución mientras que piensan de forma serena y segura.

Asimismo, los resultados permitieron evidenciar que no existe una diferencia estadística significativas entre las opiniones por edad o semestre que cursa; aunque se aprecia que las mujeres poseen una mejor percepción sobre la asignatura respecto a los hombres.

\section{REFERENCIAS BIBLIOGRÁFICAS}

Alzina, R. B. (2005). La educación emocional en la formación del profesorado. Revista interuniversitaria de formación del profesorado, (54), 95-114.

Auzmendi, E. (1992). Las actitudes hacia la matemática-estadística en la enseñanzas medias y universitarias. Bilbao, España: Mensajero.

Barbero, M.,Holgado, F., Vila, E. \& Chacón, S. (2007). Actitudes, hábitos de estudio y rendimiento en matemáticas: diferencias por género. Psicothema, 19(3), 413-421.

Cejudo Prado, J., López Delgado, M. L., Rubio Roldán, M. J., \& Latorre Postigo, J. M. (2015). La formación en educación emocional de los docentes: una visión de los futuros maestros. Revista Española de Orientación y Psicopedagogía (REOP), 26(3), 45-62. 
Cochran-Smith, M. (2003). Sometimes it's not about the Money: teaching and heart. Journal of Teacher Education, 55(4), 295-299.

Castro, Emilio J., \& Miranda, Isaías. (2019). Experiencias Desmotivacionales y Motivacionales de Estudiantes Varones de Ingeniería para Estudiar Matemáticas. EICaso de la Universidad Andrés Bello en Santiago de Chile. Formación universitaria, 12(6), 83-92. https://dx.doi.org/10.4067/S071850062019000600083

Costillo Borrego, E., Borrachero Cortés, A. B., Brígido Mero, M., \& Mellado Jiménez, V. (2013). Las emociones sobre la enseñanzaaprendizaje de las ciencias y las matemáticas de futuros profesores de Secundaria. Revista Eureka sobre Enseñanza y Divulgación de las Ciencias, 10, 514-532

De Nicolás, M. A., Torremorell, M. C. B., \& Valls, M. M. P. (2016). Dificultades en conceptos matemáticos básicos de los estudiantes para maestro. Revista INFAD de Psicología. International Journal of Developmental and Educational Psychology., 1(1), 419-430.

Domínguez, M. R. F., Pescador, J. E. P., \& Melero, M. P. T. (2009). El desarrollo socioafectivo en la formación inicial de los maestros. Revista electrónica interuniversitaria de formación del profesorado, 12(1), 2.

Fernández-Cézar, R.; Solano-Pinto, N.; Rizzo, K.; Gomezescobar-Camino, A.; Iglesias, L. M.; Espinosa, A. (2016). Las actitudes hacia las matemáticas en estudiantes y maestros de educación infantil y primaria: revisión de la adecuación de una escala para su medida. Revista Iberoamericana de Ciencia, Tecnología y Sociedad-CTS, 11(33), 227238.
Frijda, N.H. (2000). The psychologists' point of view. En: M. Lewis. y J.M. Haviland-Jones. (eds.) Handbook of emotions. New York: The Guilford Press.

Gil, N., Blanco, L. J., \& Guerrero, E. (2006). El papel de la afectividad en la resolución de problemas matemáticos. Revista de Educación, 340, p. 551-569.

Gamboa, R. (2007). Uso de la tecnología en la enseñanza de las matemáticas. Cuadernos de investigación y formación en educación matemática, 2(3), 11-44. Recuperado de https://revistas.ucr.ac.cr/index.php/cifem/ article/view/6890/657c

Gil, N., Blanco, L., \& Guerrero, E. (2005). El dominio afectivo en el aprendizaje de las matemáticas. Una revisión de sus descriptores básicos. Revista iberoamericana de educación matemática, 2(1), 15-32.

Godino, J. D., Batanero, C., \& Font, V. (2003). Fundamentos de la enseñanza y el aprendizaje de las matemáticas para maestros. Granada, España: Universidad de Granada.

Gómez-Chacón, I. M. (1997). La alfabetización emocional en educación matemática: actitudes, emociones y creencias. Uno: Revista de didáctica de las matemáticas, 13, p. 7-22.

Gómez-Chacón, I. M. (2000). Matemática Emocional. Los afectos en el aprendizaje matemático. Madrid, España: Narcea

Gómez-Chacón, I. M. (2003). La tarea intelectual en matemáticas afecto, meta-afecto y los sistemas de creencias. Boletín de la Asociación Matemática Venezolana, 10(2), p. 225-247. 
González-Pineda, J. A., \& Álvarez, L. (1998). Dificultades específicas relacionadas con las matemáticas. JL González-Pineda y J. Núñez (Coords.): Dificultades del aprendizaje escolar. Madrid: Pirámide.

Hargreaves, A. (2000). Mixed emotions: Teachers perceptions of their interactions with students. Teaching and Teacher Education, 16(7), 811-826.

Kilpatrick, J., Gómez, P., \& Rico, L. (1998). Educación matemática. Errores y dificultades de los estudiantes. Resolución de problemas. Evaluación. Historia. Una empresa docente.

Marina, J.A. (2004). La inteligencia fracasada. Barcelona, España: Anagrama.

Martínez Padrón, O. J. (2005). Dominio afectivo en educación matemática. Paradigma, 2, 7-34.

Martínez Padrón, O. J. (2014). Sistema de creencias acerca de la matemática. Revista Electrónica" Actualidades Investigativas en Educación", 14(3), 1-28. Recuperado de https://www.scielo.sa.cr/pdf/aie/v14n3/ a03v14n3.pdf

McLeod, D. B. (1989). Beliefs, attitudes, and emotions: New views of affect in mathematics education. In Affect and mathematical problem solving. New York, USA: Springer,

McLeod, D.B. (1992). Research on affect in mathematics education: $A$ reconceptualization. En D.A. Grouws (Ed.), Handbook of Research on mathematics Teaching and Learning (pp. 575-598). New York: Macmillan. USA

McLeod, D.B. (1994). Research on affect and mathematics learning in the JRME: 1970 to the present. Journal for Research in Mathematics Education, 25(6), p. 637-647.
Melero, M. P. T. (2000). La inteligencia emocional en el currículo de la formación inicial de los maestros. Revista Interuniversitaria de Formación del profesorado, (38), 141-152.

Orrantia, J. (2006). Dificultadees de aprendizaje de las matemáticas: una perspectiva evolutiva.

Revista psicopdagogía, 23(71), 158-180

Otero, M.R. (2006). Emociones, sentimientos y razonamientos en didáctica de las ciencias. Revista Electrónica de Investigación en Educación en Ciencias, 1(1).

Pichardo, I. M. C., \& Puente, Á. P. (2012). Innovación Educativa: Uso de las TIC en la enseñanza de la Matemática Básica. Edmetic, 1(2), 127-144.

Prada-Núñez, R.; Gamboa-Suárez, A. \& Avendaño-Castro, W. (2020). Caracterización del dominio afectivo hacia las matemáticas en estudiantes que ingresan a la educación superior. Revista Espacios, 41(23). Recuperado de: https://www.revistaespacios. com/a20v41n23/20412330.html

Prada Núñez, R., Gamboa Suárez, A.A. \& Hernández Suárez, C.A. (2021). Efectos depresivos del aislamiento preventivo obligatorio asociados a la pandemia del Covid-19 en docentes y estudiantes de una universidad pública en Colombia. Psicogente, 24(45), 1-20. Doi: https://doi.org/10.17081/ psico.24.45.4156

Prada-Núñez, R.; Marmolejo, G. \& Mulema, S. (2020). Belief system toward mathematics present in future teachers. A comparative study. Revista Espacios, 41(35). Recuperado: https://www.revistaespacios. com/a20v41n35/20413516.html

Rebollo, M. A., \& Rodríguez, A. L. (2006). Dificultades en el aprendizaje de las matemáticas. Revista de neurología, 135-138. DOI: https://doi.org/10.33588/rn.42S02.2005787 
Rico, L. (1995). Errores y dificultades en el aprendizaje de las matemáticas. Recuperado de http://funes.uniandes.edu.co/486/1/ RicoL95-100.PDF

Ramírez, M. D. R. R., \& Castillo, H. I. O. (2020). Funciones cognitivas y motivación en el aprendizaje de las matemáticas. Naturaleza y Tecnología, (2).

Ritchie, S.M.; Tobin, K.; Hudson, P.; Roth, W.-M. y Mergard, V. (2011). Reproducing Successful Rituals in Bad Times: Exploring Emotional Interactions of a New Science Teacher. Science Education, 94(2), 745-765.

Riviere, A. (1990). Problemas y dificultades en el aprendizaje de las matemáticas: una perspectiva cognitiva. Desarrollo psicológico y educación (Ed.), 3, 155.

Socas, Martín (2007). Dificultades y errores en el aprendizaje de las matemáticas. Análisis desde el enfoque lógico semiótico. En: Camacho, Matías; Flores, Pablo; Bolea, María Pilar (Eds.), Investigación en educación matemática (pp. 19-52). San Cristóbal de la Laguna, Tenerife: Sociedad Española de Investigación en Educación Matemática, SEIEM.

Sutton, R. \& Wheatley, K. (2003). Teachers' emotions and teaching: A review of the literature.and directions for future research. Educational Psychology Review, 15, 327358.

Vargas, N. A. V., Vega, J. A. N., \& Morales, F. H. F. (2020). Aprendizaje basado en proyectos mediados por tic para superar dificultades en el aprendizaje de operaciones básicas matemáticas. Boletín Redipe, 9(3), 167-180.

Velazquez, R., Maldonado Zúñiga, K., Del Valle Holguín, W., \& Valdéz Tamayo, P. (2020). Motivación de los estudiantes hacia el uso de la tecnología para el aprendizaje de las matemáticas. Revista Científica Sinapsis, 1(16). https://doi.org/10.37117/s. v1i16.246. 